\title{
Cost Effectiveness of Avelumab for Metastatic Merkel Cell Carcinoma
}

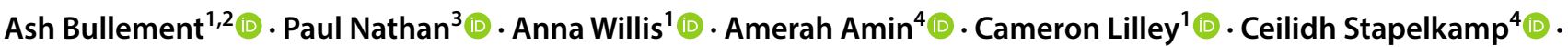 \\ Anthony Hatswell ${ }^{2,5}$ (D) Chris Pescott ${ }^{6}$ (D) Murtuza Bharmal $^{6}(\mathbb{D}$
}

Published online: 24 January 2019

(c) The Author(s) 2019

\begin{abstract}
Background Metastatic Merkel cell carcinoma (mMCC) is a rare and aggressive skin cancer. Until recently, there were no licensed treatment options for patients with $\mathrm{mMCC}$, and prognosis was poor. A cost-effectiveness analysis was conducted for avelumab, a newly available treatment option for $\mathrm{mMCC}$, versus standard care (SC), from a UK National Health Service perspective.

Methods A partitioned survival model was developed to assess the lifetime costs and effects of avelumab versus SC. Data from the JAVELIN Merkel 200 trial (NCT02155647) were used to inform estimates of quality-adjusted life-years (QALYs). Unit costs and associated frequencies of use were informed by published literature and clinical expert opinion. Results were presented as incremental cost-effectiveness ratios (ICERs, i.e. the cost per QALY gained) for treatment-experienced (TE) and treatment-naïve (TN) patients. Uncertainty was explored through a range of sensitivity analyses.

Results Discounting costs and QALYs at 3.5\% per annum, avelumab was associated with ICERs of $£ 35,274$ (TE)/£39,178 (TN) per QALY gained. Probabilistic sensitivity analysis results demonstrated that avelumab was associated with an $88.3 \%$ (TE)/69.3\% (TN) probability of being cost effective at a willingness-to-pay threshold for end-of-life treatments of £50,000 per QALY gained. Results were most sensitive to alternative survival extrapolations and dosing assumptions.

Conclusions The analysis results suggest that avelumab is likely to be a cost-effective treatment option for UK mMCC patients. The results for TN patients are subject to some uncertainty, and a confirmatory analysis will be conducted with more mature data.
\end{abstract}

\section{Key Points for Decision Makers}

Treatment options for patients with metastatic Merkel cell carcinoma (mMCC) are severely limited, and survival for patients with $\mathrm{mMCC}$ is poor with existing, unlicensed palliative chemotherapy regimens and best supportive care.

Avelumab may provide a cost-effective option for treatment-experienced mMCC patients and, while data are still maturing, demonstrates promising outcomes in treatment-naïve mMCC patients.

\section{Introduction}

Merkel cell carcinoma (MCC) is a rare, aggressive skin cancer [1-3]. It is most common in fair-skinned patients $>65$ years of age on sun-exposed skin [3, 4]. From 1999 to 2008, 1515 cases of MCC were captured by the National Cancer Data Repository in England, with a currently estimated incidence rate of $0.2-0.4$ cases per 100,000 people per year in Europe [4, 5]. MCC is associated with a high risk of local recurrence and distant metastases and is often asymptomatic on presentation, delaying diagnosis [5, 6]. The majority of MCC patients present with local or nodal disease, however an estimated $5-12 \%$ of patients present with metastatic disease $[6,7]$, and approximately $37 \%$ of

\footnotetext{
4 Merck Serono, Feltham, UK

5 Department of Statistical Science, University College London, London, UK

6 Merck KGaA, Darmstadt, Germany
} 
patients will develop metastases over the course of their disease [8-10].

Until recently, no treatment options with regulatory approval existed for patients with metastatic MCC (mMCC). Consequently, patients were typically treated with unlicensed standard care (SC): a combination of palliative chemotherapy, radiotherapy and best supportive care (BSC) $[5$, 11]. Although these treatments can induce clinically meaningful responses, responses are generally short-lived. Given the lack of an effective and well-tolerated treatment option, mMCC was associated with poor prognosis. In observational studies, estimated median survival following SC treatment is between 4 and 13 months [8, 9, 12, 13].

Avelumab (tradename: Bavencio ${ }^{\circledR}$ ) is a human immunoglobulin (Ig) G1 monoclonal antibody that targets cancer cells through the inhibition of the immune checkpoint protein programmed death-ligand 1 (PD-L1) [14]. The efficacy and safety of avelumab was studied in the pivotal phase II, single-arm JAVELIN Merkel 200 trial. Data from this trial are from two distinct cohorts: Part A, 88 treatment-experienced (TE) patients (patients who have received at least one prior line of systemic therapy for mMCC); and Part B, with a planned enrolment of 112 treatment-naïve (TN) patients [7, 15]. The findings from JAVELIN Merkel 200 demonstrate that avelumab provides an effective and well-tolerated treatment for patients with mMCC $[7,16]$.

In 2017, the National Institute for Health and Care Excellence (NICE) initiated its assessment of avelumab for mMCC (TA517) with the objective of appraising the clinical and cost effectiveness of avelumab within its marketing authorisation [17]. NICE published its final guidance for TA517 in March 2018, recommending avelumab for routine use in the National Health Service (NHS) for TE patients, and for use within the Cancer Drugs Fund for TN patients, based on preliminary data [18].

The cost-effectiveness analysis (CEA) submitted for TA517 represents the first CEA in MCC appraised by NICE.
Since publication, additional data have been made available from the JAVELIN Merkel 200 trial [19]. These data permit an update of the survival and treatment duration projections included within the CEA, acknowledged as key drivers of avelumab's estimated cost effectiveness during TA517. In this study, we provide a description of the economic model and associated inputs used to inform TA517, and update the results with the latest available data from the JAVELIN Merkel 200 trial.

\section{Methods}

\subsection{Model Overview}

A three-state, partitioned survival model was constructed to assess the cost effectiveness of avelumab versus SC. This model structure is commonly used to assess the cost effectiveness of end-stage cancer interventions, including other immune checkpoint inhibitors [20-23]. Health states considered in the model to inform estimates of costs and health-related quality of life (HRQL) were based on progression status (i.e. pre- or post-progression) and/or the time until death (categorised by $>100,30-100$, or $<30$ days until death). Transitions between progression-based health states were informed by extrapolated overall survival (OS) and progression-free survival (PFS) curves, and the time to death categorisation was estimated using OS data alone. The model structure is presented in Fig. 1.

The outcomes of interest from the model are the differences in modelled costs, life-years (LYs) and qualityadjusted life-years (QALYs) between treatment arms. Determination of the differences in costs and QALYs across the treatment arms allowed for the calculation of the cost per QALY gained, also known as the incremental cost-effectiveness ratio (ICER).
Fig. 1 Model schematic. $O S$ overall survival, $P F S$ progression-free survival
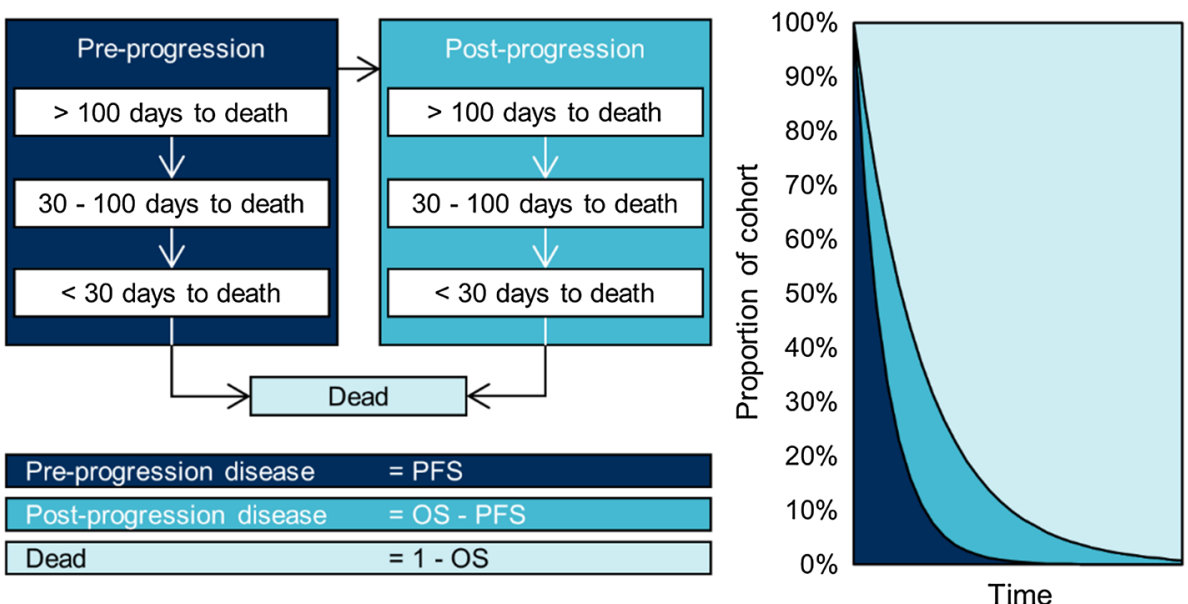
Aligned with NICE guidance, costs and QALYs were discounted at an annual rate of $3.5 \%$ and considered from a UK NHS and Personal Social Services perspective [24]. A lifetime horizon (40 years) was adopted, with a cycle length of 7 days. The time horizon was long enough to ensure that nearly $100 \%$ of (primarily elderly) patients had died by the end of the model, while the cycle length of 7 days was short enough to accurately model costs and outcomes. A cost year of 2015-2016 was used to inform the analysis, based on the latest available cost data at the time of submission to NICE.

The clinical and cost model parameters are provided in Appendix Table 3, with further details provided in Sects. 2.2 and 2.3, respectively. The validation exercises undertaken for the clinical model parameters are discussed in Sect. 2.4, and the analyses undertaken are discussed in Sect. 2.5.

\subsection{Clinical Model Parameters}

\subsubsection{Comparator Treatments}

As avelumab is the only treatment option currently licensed for patients with $\mathrm{mMCC}$, clinical expert opinion was sought to establish the component SC treatments for mMCC patients in the absence of avelumab. An overview of how clinical expert opinion was obtained is discussed in Sect. 2.4.

In the UK, SC for mMCC patients is composed of palliative chemotherapy, radiotherapy and BSC. Clinical expert opinion suggested that all chemotherapy regimens offered to patients are expected to be similarly efficacious, therefore the CEA assumed no difference in chemotherapy regimen efficacy. Furthermore, as no studies of outcomes for patients treated with BSC or individual chemotherapy regimens were identified, we assumed equivalent efficacy of all palliative chemotherapy regimens and BSC (considered appropriate by clinical experts, particularly in the TE setting).

In practice, the majority of TE patients (95\%) are expected to receive BSC as opposed to a further line of chemotherapy, whereas for TN patients approximately $50 \%$ are expected to be treated with chemotherapy, with the remainder treated with BSC. The chemotherapy regimens offered to patients with mMCC are largely platinum-based, therefore we assumed $50 \%$ of patients receiving chemotherapy are treated with carboplatin + etoposide and the remaining $50 \%$ are treated with cisplatin + etoposide (informed by clinical experts, owing to a lack of robust market share data for UK patients treated in practice) [25].

The results of this CEA considered three separate comparator treatment arms: (1) BSC; (2) chemotherapy; and (3) SC - a blend of BSC and chemotherapy aligned with clinical expert opinion. Pairwise comparisons were provided for each comparator versus avelumab. However, the base-case comparison of avelumab versus SC was considered to be the most relevant to current practice.

\subsubsection{Survival Outcomes for Comparator Treatments}

JAVELIN Merkel 200 is a single-arm trial of avelumab for patients with mMCC. Therefore, to inform the CEA, data regarding the outcomes for patients treated with palliative chemotherapy or BSC were sought. Individual-level data were available from three observational studies $(n=20 \mathrm{TE}$ US patients; $n=67$ US TN patients; and $n=34$ TE European patients) [26-28]. Aggregate-level data were reported in other published sources $[8,9,12,13,29,30]$.

Owing to similarities in patient characteristics and the designs of JAVELIN Merkel 200 and the observational studies (each conducted by Merck KGaA, Darmstadt, Germany), as well as the availability of individual-level data, the three observational studies were used to inform the OS and PFS estimates for both palliative chemotherapy and BSC. Data for TE patients were reported in two of the three studies and were naïvely pooled (pooled without adjustment for baseline characteristics) ahead of fitting parametric survival models (PSMs). Naïve pooling was undertaken as no explanatory variables (beyond whether a patient had been previously treated) could be identified using either multivariate or univariate regression, or visual inspection of Kaplan-Meier curves. Details of the analyses undertaken (and data used) have been reported by Hatswell et al. and within the NICE single technology appraisal (STA) documentation [31-33].

The Kaplan-Meier curves for the OS and PFS of TE and TN patients receiving palliative chemotherapy are provided in Fig. 2. Technical guidance from the NICE Decision Support Unit (DSU) [Technical Support Document (TSD) 14] [34] was followed when fitting PSMs. PSMs were selected based on their visual fit, statistical fit, and plausibility of their extrapolations in comparison to available external data. Gompertz and Weibull models were selected to model OS and PFS for TE patients, respectively, while log-logistic models were selected to model both OS and PFS for TN patients.

\subsubsection{Survival Outcomes for Avelumab}

OS and PFS data for patients treated with avelumab are available from the JAVELIN Merkel 200 trial. The minimum follow-up for Part A was 18 months, however data from Part B are less mature (39 of the planned 112 patients enrolled; minimum, maximum and median follow-up of 0.3, 11.3 and 5.1 months, respectively). Therefore, the survival extrapolations considered for both cohorts use data from Part A of the trial [19].

The Kaplan-Meier curves for the OS and PFS of TE and $\mathrm{TN}$ patients receiving avelumab are provided in Fig. 3 . 
Fig. 2 Survival outcomes for patients treated with palliative chemotherapy and best supportive care. $l L$ treatment-naïve, $2 L+$ treatment-experienced, $O S$ overall survival, $P F S$ progression-free survival

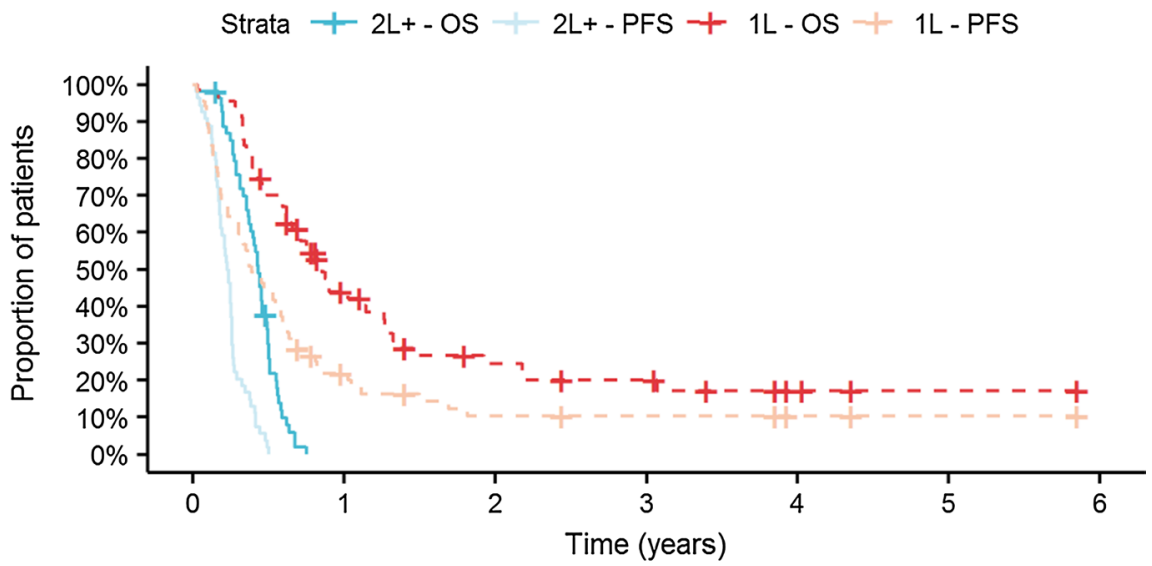

Number at risk

\begin{tabular}{|c|c|c|c|c|c|c|c|}
\hline $2 L+-O S-$ & 54 & 0 & 0 & 0 & 0 & 0 & 0 \\
\hline $2 L+-P F S-$ & 54 & 0 & 0 & 0 & 0 & 0 & C \\
\hline $1 \mathrm{~L}-\mathrm{OS}-$ & 67 & 24 & 11 & 8 & 3 & 1 & c \\
\hline $1 \mathrm{~L}-\mathrm{PFS}-$ & 67 & 12 & 5 & 4 & 2 & 1 & c \\
\hline & 1 & 1 & $\begin{array}{l}7 \\
2\end{array}$ & 3 & 4 & 5 & 6 \\
\hline
\end{tabular}

Owing to the plateau observed in the PFS curve for TE patients from approximately 6 months, as well as the emergent plateau in the OS curve, flexible PSMs were explored to inform the estimation of OS and PFS in the longer term for TE patients treated with avelumab. Royston and Parmar natural cubic spline-based models were applied for the OS and PFS of patients treated with avelumab in the CEA base case [35]. The spline-based models exhibit greater sensitivity compared with traditional PSMs, to reflect the changing hazards in the observed survival in JAVELIN Merkel 200.

For TN patients, data were considered too immature to extrapolate into the longer term, therefore two distinct approaches were taken to model survival-based outcomes for this cohort: (1) use expert-elicited hazard ratios (HRs) via a
Fig. 3 Survival outcomes for patients treated with avelumab. Data-cut for Part A: 26 September 2017; data-cut for Part B: 24 March 2017. $1 L$ treatmentnaïve, $2 L+$ treatment-experienced, $O S$ overall survival, $P F S$ progression-free survival

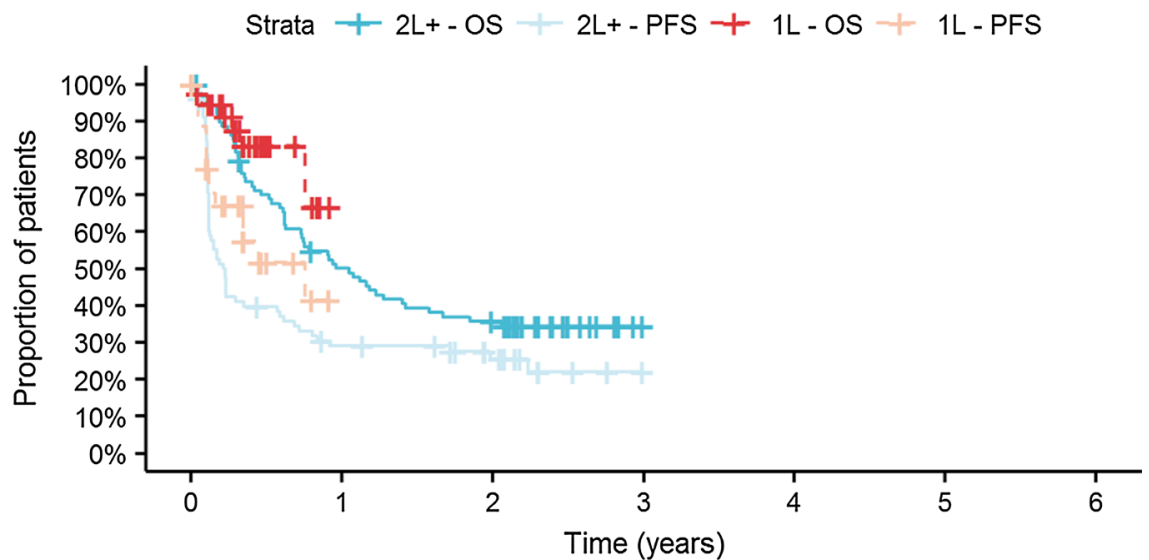

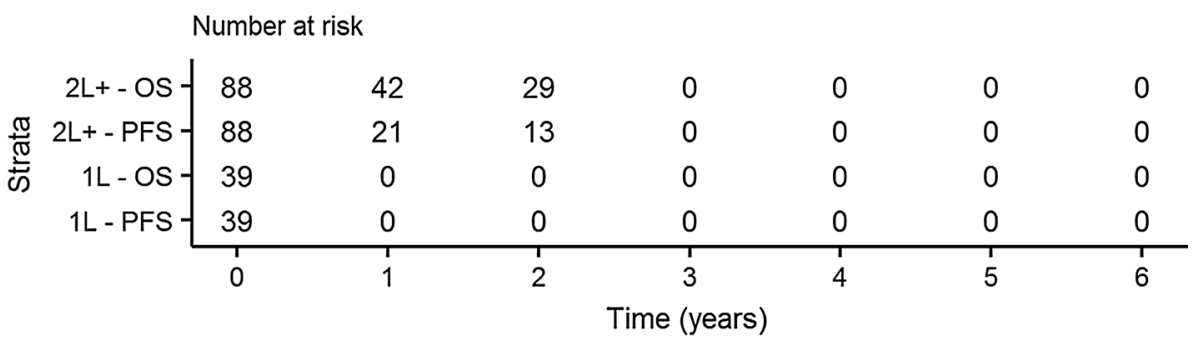


face-to-face interview to adjust the OS (0.8) and PFS (1.0) extrapolations for TE patients based on the expected increase in average survival for TN patients versus TE patients; or (2) use a curve fitted to the TN data until no longer considered valid, and then consider the extrapolation for TE patients. In the model base case, we used approach 1 as this method synthesises mature data for TE patients in the longer term with clinical expert opinion regarding the expected improvement in OS in the shorter term, instead of relying on the maturing Part B data alone. However, approach 2 was considered as a scenario analysis.

\subsubsection{Adverse Events}

The CEA also included the cost and/or health impacts of any treatment-emergent adverse events (AEs). AE rates for patients treated with palliative chemotherapy were sourced from a systematic literature review, where one study was used for each chemotherapy regimen considered (in smallcell lung cancer [SCLC], as no evidence was available for MCC and clinical expert opinion considered SCLC a reasonable proxy in the absence of data for MCC) [25, 36, 37]. For unreported AEs for the palliative chemotherapy regimens, a rate of $0 \%$ was applied conservatively.

For avelumab patients, AE rates were taken from Part A of the JAVELIN Merkel 200 trial (due to the maturity of data from this cohort) and assumed to apply for both TE and $\mathrm{TN}$ patients. Overall, avelumab was well tolerated compared with chemotherapy regimens, which, in general, are associated with high rates of haematological abnormalities and hair loss [7, 36, 37]. The most commonly reported AEs for avelumab and chemotherapy were lymphopenia (grade $\geq 3$, $2.27 \%$ ) and neutropenia (grade $\geq 3,45.49 \%$ ), respectively $[7,36,37]$.

\subsubsection{Health-Related Quality of Life}

HRQL was assessed for patients in JAVELIN Merkel 200 at baseline, week 7, every 6 weeks thereafter, and at the endof-treatment visit via the EQ-5D-5L ${ }^{\circledR}$ questionnaire $[38,39]$. Data from Part A of the JAVELIN Merkel 200 trial were analysed to inform the CEA for both cohorts of patients.

Two approaches to incorporating HRQL data were considered in the model: (1) a time-to-death approach using the 'crosswalk' algorithm reported by van Hout et al. to convert EQ-5D-5L to EQ-5D-3L ${ }^{\circledR}$ values; and (2) a progressionbased approach using the EQ-5D-5L value set reported by Devlin et al. [31, 33, 39-42]. The CEA base case was informed by time-to-death utilities used in the NICE STA, and the progression-based approach was considered as a scenario analysis. The time-to-death approach was used in the NICE STA as few measurements of EQ-5D-5L were available for patients beyond progression in JAVELIN Merkel
200. Furthermore, measurements were collected solely for patients who were fit enough to continue treatment after progression. Therefore, the use of progression-based utilities may overestimate the HRQL of progressed patients in clinical practice.

Disutilities for AEs were also included for patients receiving active treatment (i.e. avelumab or palliative chemotherapy), with disutility values taken from published sources [43-45]. Disutilities attributable to AEs are applied as a weekly QALY decrement to those patients receiving treatment.

\subsection{Cost Model Parameters}

\subsubsection{Treatment Costs}

Avelumab is available in a $200 \mathrm{mg} / 10 \mathrm{~mL}$ vial priced at $£ 768.00$ [46]. Per its summary of product characteristics, avelumab is administered as an intravenous infusion once every 2 weeks at a target dose of $10 \mathrm{mg} / \mathrm{kg}$ [14]. To determine the average cost per administration, the distribution of patient weight was determined from patients in Part A of the JAVELIN Merkel 200 trial, and the relative dose intensity of $95.4 \%$ observed within the trial was accounted for [47].

After fitting a log-normal distribution to the weight data, the proportion of patients requiring each number of vials per administration was determined and costed within the model. This method ensures drug wastage is included within the avelumab costing (e.g. a $79 \mathrm{~kg}$ patient requires $790 \mathrm{mg}$ and would therefore incur the cost of four vials [10 $\mathrm{mg}$ wastage], whereas an $81 \mathrm{~kg}$ patient requires $810 \mathrm{mg}$ and would therefore incur the cost of five vials [ $190 \mathrm{mg}$ wastage]). This technique for costing weight-based treatments is often termed the 'method of moments' [48].

In the model base case, the 'method of moments' approach was applied for European patients as this method costs all patients per the exact licensed dose and considers the group of patients expected to be most similar to those treated in NHS practice. This results in an average of 4.25 vials per administration, costing $£ 3261.04$. A scenario analysis was considered using the published NHS England National Dose Banding Table (NDBT) for avelumab [49].

Time on treatment (ToT) data were taken from Part A of the JAVELIN Merkel 200 trial to inform the duration of treatment. Clinical expert opinion suggested that based on the precedent set by other immune checkpoint inhibitors, the majority of patients (i.e. approximately $95 \%$ of those initiating treatment) would not be expected to receive avelumab after 2 years, and all patients are likely to have discontinued by 5 years. A Weibull PSM provided the best fit to the observed data and aligned with the aforementioned clinical expert opinion. For TN patients, an HR of 1.0 was used to inform the model base case (per clinical expert opinion); 
however, in a scenario analysis, the immature $\mathrm{TN}$ data were used as the basis for extrapolation. Like TE patients, clinicians advised that at 2 years, only $5 \%$ of patients were assumed to continue treatment, and by 5 years all patients were assumed to have discontinued.

Carboplatin + etoposide and cisplatin + etoposide were administered according to their use in previous studies of SCLC [36, 37]. During validation through consultation with mMCC clinical experts, it was suggested that for the carboplatin + etoposide regimen, patients could take an oral dose of etoposide $200 \mathrm{mg} / \mathrm{m}^{2}$ for days 2 and 3 of a 3-week cycle $[25,36,37]$. This regimen was assumed in the basecase CEA. Both chemotherapy regimens were assumed to be administered for a maximum of six treatment cycles (18 weeks) unless a patient progressed or died.

Unit costs for the chemotherapy regimens were sourced from the British National Formulary (BNF) for branded medicines and the NHS electronic market information tool (eMIT) for generic medicines associated with published NHS discounts. Using a clinically validated estimate of the relative dose intensity for palliative chemotherapy (66.7\%) and an assumed 50:50 split across both regimens, a weekly cost of $£ 12.43$ was applied in the CEA for the cost of chemotherapy treatment.

Treatments costed within the model are administered either as an intravenous or oral treatment. An intravenous administration cost of $£ 199$, taken from the NHS reference costs database, was applied per intravenous administration, whereas the cost of oral medication administration was assumed to be zero [50].

\subsubsection{Medical Resource Use}

Patients are expected to incur a range of monitoring costs, including general practitioner (GP) visits, computed tomography (CT) scans, and routine blood and organ function tests. Costs of monitoring and resource utilisation were taken from the NHS reference costs database and the Unit Costs for Health and Social Care [50, 51]. Frequencies of monitoring visits and tests were procured from $\mathrm{mMCC}$ clinical experts.

In addition to the costs of monitoring, some patients may receive palliative radiotherapy for symptom management. Consultation with mMCC clinical experts suggested that radiation therapy is received by approximately $75 \%$ of patients, involving approximately one to five fractions, and administered one to two times in total. The cost per fraction of radiotherapy treatment was taken from the NHS reference costs database [50].

Costs associated with AE resolution were identified from published literature sources and were inflated using the Hospital and Community Health Services (HCHS) index reported in the Unit Costs for Health and Social Care [51-53].
As no data were identified regarding the costs associated with end-of-life care for $\mathrm{mMCC}$ patients, average costs for health and social care relating to end-of-life care for terminal cancer patients were taken from the study by Round et al. [54]. The costs reported in that study were inflated using the aforementioned HCHS index [51]. The total cost for end-oflife care was $£ 7019.12$, applied within the CEA as a lump sum upon death.

\subsection{Clinical and External Validation}

Inputs considered within the CEA were validated by mMCC clinical experts through an advisory board and follow-up one-to-one consultations [25]. Consultation with clinical experts led to the inclusion of radiotherapy costs for symptom management and confirmation of the standard chemotherapy regimens considered (platinum-based chemotherapy), as well as the relative use of carboplatin and cisplatin (cisplatin is used for patients unable to tolerate carboplatin). The choice of model structure, survival extrapolation techniques and approaches to incorporating HRQL data were also validated by both clinical and economic experts.

The submitted CEA, as well as later updates, were critiqued by an independent Evidence Review Group (ERG) during NICE TA517 [31]. The ERG's comments were implemented within the revised model, including the use of a Weibull curve for the avelumab ToT curve, inflation of endof-life care costs, and the appropriate frequencies of medical resource use [55]. Finally, the CEA was subject to several independent quality control checks throughout development to check for modelling errors and/or implausible results by stress testing various model inputs and assumptions.

\subsection{Analysis}

The base case considered the results of a deterministic CEA. Probabilistic sensitivity analysis (PSA) was conducted to ascertain the probability of avelumab being a cost-effective treatment option for mMCC patients, at the willingness-topay threshold $(\lambda)$ of $£ 50,000$ considered cost effective for end-of-life treatments [56]. Deterministic scenario analysis (DSA) was also undertaken to assess the sensitivity of the base-case results to various structural and/or alternative assumptions. The scenarios considered alternative assumptions regarding survival extrapolation methods, use of TE data to inform TN patient outcomes, application of HRQL data, and dosing. 


\section{Results}

\subsection{Base-Case Results}

The base-case CEA results are provided in Table 1. At a $\lambda$ of $£ 50,000$ per QALY gained, avelumab was shown to be a cost-effective treatment option for TE and TN mMCC patients. The base-case ICER was $£ 35,274$ for the TE population and $£ 39,178$ for the TN population. Avelumab was estimated to provide 45 months extension to OS for TE patients and 43 months extension to OS for TN patients compared with SC. Furthermore, an increase of 2.24 and 1.96 QALYs was predicted for avelumab versus SC for the TE and TN populations, respectively.

\subsection{Probabilistic Sensitivity Analysis}

PSA was performed by running 2000 probabilistic iterations for each modelled population of mMCC patients. The results of the PSA (presented in Table 2 and the Appendix Table 3) demonstrated that avelumab was associated with an $88.3 \%$ (TE)/69.3\% (TN) probability of being cost effective at a $\lambda$ of $£ 50,000$ per QALY gained versus SC (Figs. 4, 5, 6, 7).

\subsection{Deterministic Sensitivity Analysis}

The DSA undertaken considered alternative assumptions regarding the application of HRQL data, the dosing of avelumab and the use of data to inform survival extrapolations for TN patients. The results of the scenarios are discussed in turn below.
Table 1 Base-case deterministic pairwise cost-effectiveness results
Table 2 Base-case probabilistic cost-effectiveness results

\begin{tabular}{|c|c|c|c|c|c|c|c|}
\hline \multirow[t]{2}{*}{ Treatment } & \multicolumn{3}{|l|}{ Total } & \multicolumn{3}{|c|}{ Incremental (vs. avelumab) } & \multirow[t]{2}{*}{ 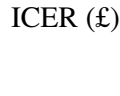 } \\
\hline & Costs $(£)$ & LYs & QALYs & Costs $(£)$ & LYs & QALYs & \\
\hline \multicolumn{8}{|l|}{$T E m M C C$} \\
\hline Chemotherapy & 9834 & 0.41 & 0.30 & 78,395 & 3.74 & 2.30 & 34,113 \\
\hline $\mathrm{SC}$ & 7584 & 0.41 & 0.31 & 80,646 & 3.74 & 2.29 & 35,274 \\
\hline BSC & 7465 & 0.41 & 0.31 & 80,764 & 3.74 & 2.29 & 35,335 \\
\hline Avelumab & 88,229 & 4.15 & 2.60 & & & & \\
\hline \multicolumn{8}{|l|}{$T N m M C C$} \\
\hline Chemotherapy & 10,607 & 1.94 & 1.34 & 77,292 & 3.56 & 2.02 & 38,205 \\
\hline $\mathrm{SC}$ & 8918 & 1.94 & 1.35 & 78,981 & 3.56 & 2.02 & 39,178 \\
\hline BSC & 7229 & 1.94 & 1.36 & 80,669 & 3.56 & 2.01 & 40,158 \\
\hline Avelumab & 87,899 & 5.50 & 3.37 & & & & \\
\hline
\end{tabular}

$B S C$ best supportive care, ICER incremental cost-effectiveness ratio, $L Y s$ life-years, $m M C C$ metastatic Merkel cell carcinoma, $Q A L Y S$ quality-adjusted life-years, $S C$ standard care, $T E$ treatment-experienced, $T N$ treatment-naïve

\begin{tabular}{|c|c|c|c|c|c|c|c|}
\hline \multicolumn{8}{|c|}{ Pairwise cost-effectiveness results } \\
\hline \multirow[t]{2}{*}{ Treatment } & \multicolumn{3}{|l|}{ Total } & \multicolumn{3}{|c|}{ Incremental (vs. avelumab) } & \multirow[t]{2}{*}{$\operatorname{ICER}(\mathfrak{f})$} \\
\hline & Costs $(£)$ & LYs & QALYs & Costs $(£)$ & LYs & QALYs & \\
\hline \multicolumn{8}{|l|}{$T E m M C C$} \\
\hline Chemotherapy & 9838 & 0.41 & 0.30 & 80,143 & 3.84 & 2.35 & 34,076 \\
\hline $\mathrm{SC}$ & 7591 & 0.41 & 0.31 & 82,390 & 3.84 & 2.34 & 35,208 \\
\hline BSC & 7473 & 0.41 & 0.31 & 82,508 & 3.84 & 2.34 & 35,268 \\
\hline Avelumab & 89,981 & 4.26 & 2.65 & & & & \\
\hline \multicolumn{8}{|l|}{$T N m M C C$} \\
\hline Chemotherapy & 10,622 & 1.95 & 1.35 & 80,573 & 3.64 & 2.07 & 38,986 \\
\hline $\mathrm{SC}$ & 8929 & 1.95 & 1.36 & 82,266 & 3.64 & 2.06 & 39,943 \\
\hline BSC & 7236 & 1.95 & 1.37 & 83,959 & 3.64 & 2.05 & 40,907 \\
\hline Avelumab & 91,194 & 5.60 & 3.42 & & & & \\
\hline
\end{tabular}

$B S C$ best supportive care, ICER incremental cost-effectiveness ratio, $L Y s$ life-years, $m M C C$ metastatic Merkel cell carcinoma, $Q A L Y S$ quality-adjusted life-years, $S C$ standard care, $T E$ treatment-experienced, $T N$ treatment-naïve 
Table 3 Model parameters

\begin{tabular}{|c|c|c|c|c|c|}
\hline Parameter & Value & $\begin{array}{l}\text { Lower } 95 \% \\
\text { CI }\end{array}$ & Upper $95 \%$ CI & Distribution & Source or justification \\
\hline Discount rate, costs & $3.50 \%$ & NA & NA & Varied in scenario analysis & NICE reference case [59] \\
\hline Discount rate, QALYs & $3.50 \%$ & NA & NA & Varied in scenario analysis & \\
\hline Discount rate, LYs & $0.00 \%$ & NA & NA & Varied in scenario analysis & \\
\hline Model cycle length & 1 week & NA & NA & Not varied & Structural assumption \\
\hline Model time horizon & 40 years & NA & NA & Not varied & NICE reference case [59] \\
\hline $\begin{array}{l}\text { HR: improvement in OS for avelumab (TN } \\
\text { vs. TE) }\end{array}$ & 0.800 & 0.655 & 0.968 & Log-normal & $\begin{array}{l}\text { Assumptions validated by } \\
\text { clinical expert opinion }\end{array}$ \\
\hline $\begin{array}{l}\text { HR: improvement in PFS for avelumab (TN } \\
\text { vs. TE) }\end{array}$ & 1.000 & 0.818 & 1.210 & Log-normal & \\
\hline RDI: avelumab & $95.43 \%$ & $93.59 \%$ & $97.27 \%$ & Normal & JAVELIN Merkel 200 [7] \\
\hline RDI: chemotherapy & $66.67 \%$ & $53.60 \%$ & $79.73 \%$ & Normal & Clinical validation \\
\hline Utility, progression-free, $(-5 \mathrm{~L})$ & 0.827 & 0.783 & 0.867 & Beta & Kaufman et al. [39] \\
\hline Utility, post-progression (-5L) & 0.742 & 0.690 & 0.790 & Beta & \\
\hline Utility, $>100$ days to death $(-3 \mathrm{~L})$ & 0.774 & 0.734 & 0.813 & Beta & NICE TA517 [55] \\
\hline Utility, 30-100 days to death (-3L) & -0.020 & 0.000 & -0.108 & Beta & \\
\hline Utility, $<30$ days to death $(-3 \mathrm{~L})$ & -0.066 & -0.020 & -0.138 & Beta & \\
\hline Administration cost, IV drugs & $£ 199.00$ & $£ 198.81$ & $£ 199.19$ & Normal & NHS reference costs $15-16$ \\
\hline Cost, GP visit & $£ 36.00$ & $£ 28.94$ & $£ 43.06$ & Normal & [50], PSSRU 2016 [51] \\
\hline Cost, CT scan & $£ 120.99$ & $£ 120.95$ & $£ 121.03$ & Normal & \\
\hline Cost, FBC & $£ 3.00$ & $£ 3.00$ & $£ 3.00$ & Normal & \\
\hline Cost, LFT & $£ 1.00$ & $£ 0.80$ & $£ 1.20$ & Normal & \\
\hline Cost, RFT & $£ 1.00$ & $£ 0.80$ & $£ 1.20$ & Normal & \\
\hline Cost, TFT & $£ 1.00$ & $£ 0.80$ & $£ 1.20$ & Normal & \\
\hline Cost, radiotherapy & $£ 126.60$ & $£ 126.58$ & $£ 126.62$ & Normal & \\
\hline Cost, EoL, health care & $£ 4867.53$ & $£ 3913.51$ & $£ 5821.55$ & Normal & Round et al. [54] \\
\hline Cost, EoL, social care & $£ 2151.59$ & $£ 1729.89$ & $£ 2573.29$ & Normal & \\
\hline MRU frequency, GP visit, avelumab, PF & 0.250 & 0.201 & 0.299 & Normal & Assumptions validated by \\
\hline MRU frequency, CT scan, avelumab, PF & 0.077 & 0.062 & 0.092 & Normal & clinical expert opinion \\
\hline MRU frequency, FBC, avelumab, PF & 0.500 & 0.402 & 0.598 & Normal & \\
\hline MRU frequency, LFT, avelumab, PF & 0.500 & 0.402 & 0.598 & Normal & \\
\hline MRU frequency, RFT, avelumab, PF & 0.500 & 0.402 & 0.598 & Normal & \\
\hline MRU frequency, TFT, avelumab, PF & 0.500 & 0.402 & 0.598 & Normal & \\
\hline $\begin{array}{l}\text { MRU frequency, radiotherapy, avelumab, } \\
\text { PF }\end{array}$ & 0.000 & 0.000 & 0.000 & Normal & \\
\hline $\begin{array}{l}\text { MRU frequency, GP visit, chemotherapy, } \\
\text { PF }\end{array}$ & 0.333 & 0.268 & 0.399 & Normal & \\
\hline $\begin{array}{l}\text { MRU frequency, CT scan, chemotherapy, } \\
\text { PF }\end{array}$ & 0.115 & 0.093 & 0.138 & Normal & \\
\hline MRU frequency, FBC, chemotherapy, PF & 0.333 & 0.268 & 0.399 & Normal & \\
\hline MRU frequency, LFT, chemotherapy, PF & 0.333 & 0.268 & 0.399 & Normal & \\
\hline MRU frequency, RFT, chemotherapy, PF & 0.333 & 0.268 & 0.399 & Normal & \\
\hline MRU frequency, TFT, chemotherapy, PF & 0.000 & 0.000 & 0.000 & Normal & \\
\hline $\begin{array}{l}\text { MRU frequency, radiotherapy, chemo- } \\
\text { therapy, PF }\end{array}$ & 0.000 & 0.000 & 0.000 & Normal & \\
\hline Drug cost, avelumab & $£ 768.00$ & NA & NA & Not varied & BNF online [46] \\
\hline Drug cost, carboplatin & $£ 25.25$ & $£ 25.18$ & $£ 25.32$ & Normal & eMIT [60] \\
\hline Drug cost, etoposide IV & $£ 24.96$ & $£ 22.16$ & $£ 27.76$ & Normal & eMIT [60] \\
\hline Drug cost, etoposide oral & $£ 87.23$ & NA & NA & Not varied & BNF online [46] \\
\hline Drug cost, cisplatin & $£ 10.56$ & $£ 10.49$ & $£ 10.63$ & Normal & eMIT [60] \\
\hline AE probability, lymphopenia, avelumab & $2.27 \%$ & & & Beta & JAVELIN Merkel 200 [7] \\
\hline
\end{tabular}


Table 3 (continued)

\begin{tabular}{|c|c|c|c|c|c|}
\hline Parameter & Value & $\begin{array}{l}\text { Lower } 95 \% \\
\text { CI }\end{array}$ & Upper 95\% CI & Distribution & Source or justification \\
\hline $\begin{array}{l}\text { AE probability, anaemia, carbopl- } \\
\text { atin + etoposide }\end{array}$ & $7.38 \%$ & & & Beta & \multirow[t]{9}{*}{ Socinski et al. [36] } \\
\hline $\begin{array}{l}\text { AE probability, fatigue, carboplatin + etopo- } \\
\text { side }\end{array}$ & $3.13 \%$ & & & Beta & \\
\hline $\begin{array}{l}\text { AE probability, febrile neutropenia, carbo- } \\
\text { platin + etoposide }\end{array}$ & $4.47 \%$ & & & Beta & \\
\hline $\begin{array}{l}\text { AE probability, hyponatraemia, carbopl- } \\
\text { atin + etoposide }\end{array}$ & $1.12 \%$ & & & Beta & \\
\hline $\begin{array}{l}\text { AE probability, leukopenia, carbopl- } \\
\text { atin + etoposide }\end{array}$ & $8.28 \%$ & & & Beta & \\
\hline $\begin{array}{l}\text { AE probability, nausea/vomiting, carbopl- } \\
\text { atin + etoposide }\end{array}$ & $0.90 \%$ & & & Beta & \\
\hline $\begin{array}{l}\text { AE probability, neutropenia, carbopl- } \\
\text { atin + etoposide }\end{array}$ & $46.98 \%$ & & & Beta & \\
\hline $\begin{array}{l}\text { AE probability, thrombocytopenia, carbopl- } \\
\text { atin + etoposide }\end{array}$ & $10.29 \%$ & & & Beta & \\
\hline $\begin{array}{l}\text { AE probability, hair loss, carbopl- } \\
\text { atin + etoposide }\end{array}$ & $34.00 \%$ & & & Beta & \\
\hline $\begin{array}{l}\text { AE probability, anaemia, cisplatin + etopo- } \\
\text { side }\end{array}$ & $6.67 \%$ & & & Beta & \multirow[t]{7}{*}{ Sun et al. [37] } \\
\hline $\begin{array}{l}\text { AE probability, low haemoglobin, cispl- } \\
\text { atin + etoposide }\end{array}$ & $5.33 \%$ & & & Beta & \\
\hline $\begin{array}{l}\text { AE probability, leukopenia, cispl- } \\
\text { atin + etoposide }\end{array}$ & $19.33 \%$ & & & Beta & \\
\hline $\begin{array}{l}\text { AE probability, nausea/vomiting, cispl- } \\
\text { atin + etoposide }\end{array}$ & $6.70 \%$ & & & Beta & \\
\hline $\begin{array}{l}\text { AE probability, neutropenia, cispl- } \\
\text { atin + etoposide }\end{array}$ & $44.00 \%$ & & & Beta & \\
\hline $\begin{array}{l}\text { AE probability, thrombocytopenia, cispl- } \\
\text { atin + etoposide }\end{array}$ & $7.33 \%$ & & & Beta & \\
\hline $\begin{array}{l}\text { AE probability, hair loss, cisplatin + etopo- } \\
\text { side }\end{array}$ & $13.33 \%$ & & & Beta & \\
\hline $\mathrm{AE}$ cost, anaemia & $£ 799.39$ & $£ 657.09$ & $£ 977.46$ & Normal & \multirow{11}{*}{$\begin{array}{l}\text { Vouk et al. [52] and Wehler } \\
\text { et al. [53] }\end{array}$} \\
\hline $\mathrm{AE}$ cost, fatigue & $£ 66.45$ & $£ 53.43$ & $£ 79.47$ & Normal & \\
\hline AE cost, febrile neutropenia & $£ 4543.44$ & $£ 3652.94$ & $£ 5433.93$ & Normal & \\
\hline AE cost, low haemoglobin & $£ 66.45$ & $£ 53.43$ & $£ 79.47$ & Normal & \\
\hline AE cost, hyponatraemia & $£ 66.45$ & $£ 53.43$ & $£ 79.47$ & Normal & \\
\hline AE cost, leukopenia & $£ 281.67$ & $£ 226.46$ & $£ 336.88$ & Normal & \\
\hline AE cost, lymphopenia & $£ 281.67$ & $£ 226.46$ & $£ 336.88$ & Normal & \\
\hline AE cost, nausea/vomiting & $£ 218.27$ & $£ 181.41$ & $£ 269.86$ & Normal & \\
\hline AE cost, neutropenia & $£ 281.67$ & $£ 226.46$ & $£ 336.88$ & Normal & \\
\hline AE cost, thrombocytopenia & $£ 286.12$ & $£ 230.05$ & $£ 342.20$ & Normal & \\
\hline $\mathrm{AE}$ cost, hair loss & $£ 0.00$ & $£ 0.00$ & $£ 0.00$ & Normal & \\
\hline AE disutility, anaemia & -0.090 & -0.055 & -0.133 & Beta & \multirow{11}{*}{$\begin{array}{l}\text { Nafees et al. [43], Ossa et al. } \\
\text { [45], Tolley et al. [44] and } \\
\text { assumptions validated by } \\
\text { clinical expert opinion }\end{array}$} \\
\hline AE disutility, fatigue & -0.073 & -0.041 & -0.114 & Beta & \\
\hline AE disutility, febrile neutropenia & -0.090 & -0.061 & -0.124 & Beta & \\
\hline AE disutility, low haemoglobin & -0.080 & -0.052 & -0.114 & Beta & \\
\hline AE disutility, hyponatraemia & -0.090 & -0.062 & -0.122 & Beta & \\
\hline AE disutility, leukopenia & -0.090 & -0.062 & -0.122 & Beta & \\
\hline AE disutility, lymphopenia & -0.090 & -0.062 & -0.122 & Beta & \\
\hline AE disutility, nausea/vomiting & -0.048 & -0.022 & -0.084 & Beta & \\
\hline AE disutility, neutropenia & -0.090 & -0.062 & -0.122 & Beta & \\
\hline AE disutility, thrombocytopenia & -0.108 & -0.089 & -0.128 & Beta & \\
\hline AE disutility, hair loss & -0.045 & -0.021 & -0.078 & Beta & \\
\hline
\end{tabular}


Table 3 (continued)

\begin{tabular}{|c|c|c|c|c|c|}
\hline Parameter & Value & $\begin{array}{l}\text { Lower } 95 \% \\
\text { CI }\end{array}$ & Upper $95 \%$ CI & Distribution & Source or justification \\
\hline AE duration, anaemia & 21 days & 17 days & 25 days & Normal & \multirow{11}{*}{$\begin{array}{l}\text { Assumptions validated by } \\
\text { clinical expert opinion }\end{array}$} \\
\hline AE duration, fatigue & 21 days & 17 days & 25 days & Normal & \\
\hline AE duration, febrile neutropenia & 4 days & 3 days & 5 days & Normal & \\
\hline AE duration, low haemoglobin & 21 days & 17 days & 25 days & Normal & \\
\hline AE duration, hyponatraemia & 2 days & 2 days & 2 days & Normal & \\
\hline AE duration, leukopenia & 2 days & 2 days & 2 days & Normal & \\
\hline AE duration, lymphopenia & 2 days & 2 days & 2 days & Normal & \\
\hline AE duration, nausea/vomiting & 3 days & 2 days & 4 days & Normal & \\
\hline AE duration, neutropenia & 2 days & 2 days & 2 days & Normal & \\
\hline AE duration, thrombocytopenia & 24 days & 19 days & 28 days & Normal & \\
\hline AE duration, hair loss & 21 days & 17 days & 25 days & Normal & \\
\hline
\end{tabular}

3L 3-level, 5L 5-level, $A E$ adverse event, $B N F$ British National Formulary, $C I$ confidence interval, $C T$ computed tomography, $e M I T$ electronic market information tool, $E o L$ end of life, $F B C$ full blood count, $G P$ general practitioner, $H R$ hazard ratio, $I V$ intravenous, $L F T$ liver function test, $L Y S$ life-years, $M R U$ medical resource use, NA not applicable, NHS National Health Service, NICE National Institute for Health and Care Excellence, $O S$ overall survival, $P F$ progression-free, $P F S$ progression-free survival, PSSRU Personal Social Services Research Unit, $Q A L Y S$ quality-adjusted life-years, $R D I$ relative dose intensity, $R F T$ renal function test, $T A$ technology appraisal, $T E$ treatment-experienced, $T F T$ thyroid function test, $T N$ treatment-naïve

When applying the progression-based utilities, the basecase ICERs for TE and TN patients decreased to $£ 33,644$ and $£ 37,494$, respectively. This was due to the relatively high PFS health-state utility value of 0.83 (using the EQ-5D-5L value set) compared with the utility value for patients with $>100$ days to death of 0.77 (using the EQ-5D-3L value set).

Applying the NDBT guidance to reflect the dosing of avelumab reduced the ICERs for TE and TN patients to $£ 32,784$ and $£ 37,849$, respectively. The NDBT for avelumab resulted in a lower average dose per administration, hence a lower cost for avelumab-treated patients.

When TN data were applied to extrapolate OS, PFS and ToT for TN patients until the hazard of death exceeded that of the extrapolation for TE patients (at approximately 21 months), and the ToT was appropriately adjusted to reflect clinical expert opinion regarding discontinuation at 2 and 5 years, the ICER increased to $£ 48,102$. This increase in the ICER was primarily driven by the extrapolation of immature ToT data.

\section{Discussion}

This study demonstrates that avelumab may be a cost-effective treatment option for UK TE or TN mMCC patients, with ICERs versus SC of $£ 35,274$ and $£ 39,182$, respectively. The survival benefits attributable to avelumab versus SC are unprecedented within the context of $\mathrm{mMCC}$, therefore avelumab provides a substantial improvement to the management of this life-threatening rare disease. A comprehensive set of sensitivity analyses further demonstrated the robustness of the base-case CEA results. The results were most sensitive to alternative survival extrapolations and treatment costing assumptions.

This study is based on a CEA considering a UK perspective, with structural features and clinical assumptions validated by clinical and economic experts. Data regarding the safety and efficacy of avelumab come from JAVELIN Merkel 200, the largest registrational trial conducted in mMCC to date. In addition to potentially prolonged survival outcomes, with estimated 12-month survival of 51\% (95\% confidence interval [CI] 40-61\%) and 18-month survival of $40 \%$ (95\% CI 29-50\%), avelumab was shown, within the JAVELIN Merkel 200 trial, to be a well-tolerated treatment option for mMCC and associated with few treatment-related AEs compared with conventional palliative chemotherapy regimens.

Key limitations of this study relate to the rarity of mMCC and, as a consequence, the adoption of the single-arm JAVELIN Merkel 200 trial design. Single-arm studies are typical for ultra-rare diseases, particularly those with an evolving treatment pathway, when numerous ongoing clinical trials are expected to impact the future treatment pathway [57]. Naïve comparisons were undertaken because of the small patient numbers in the relevant studies. While adjusted comparisons were attempted (not reported in this study, but reported within NICE documentation), these were limited due to both sample sizes and the reporting of relevant patient characteristics, and were not greatly influential on cost-effectiveness results [55]. As long-term data from JAVELIN Merkel 200 become available, the uncertainty regarding the long-term survival outcomes for patients treated with avelumab will reduce; however, due to the rarity of MCC and limited treatment options, standard 


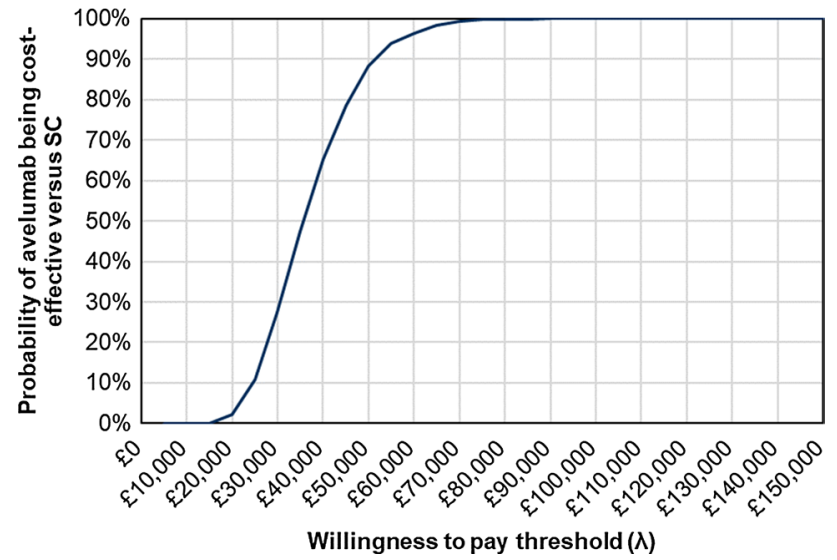

Fig. 4 Cost-effectiveness acceptability curve versus standard care, treatment-experienced mMCC patients. $m M C C$ metastatic Merkel cell carcinoma, $S C$ standard care

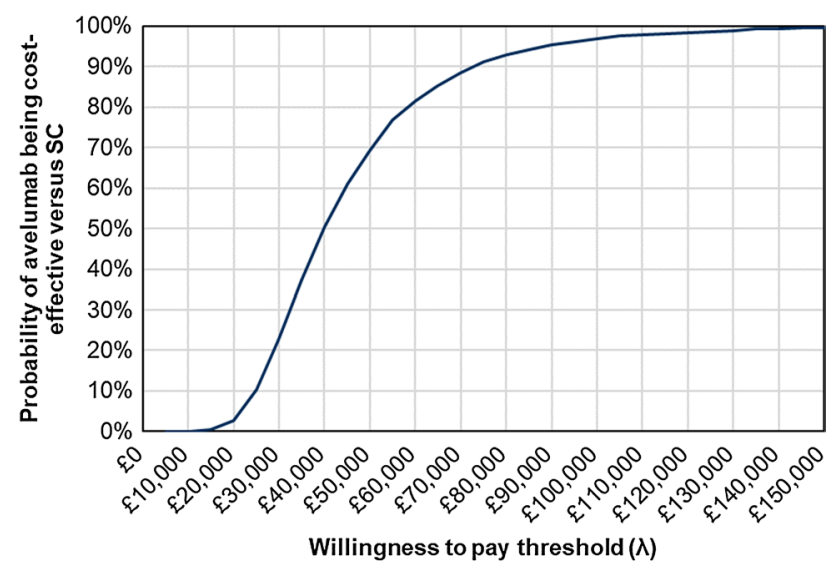

Fig. 5 Cost-effectiveness acceptability curve versus standard care, treatment-naive mMCC patients. $m M C C$ metastatic Merkel cell carcinoma, $S C$ standard care

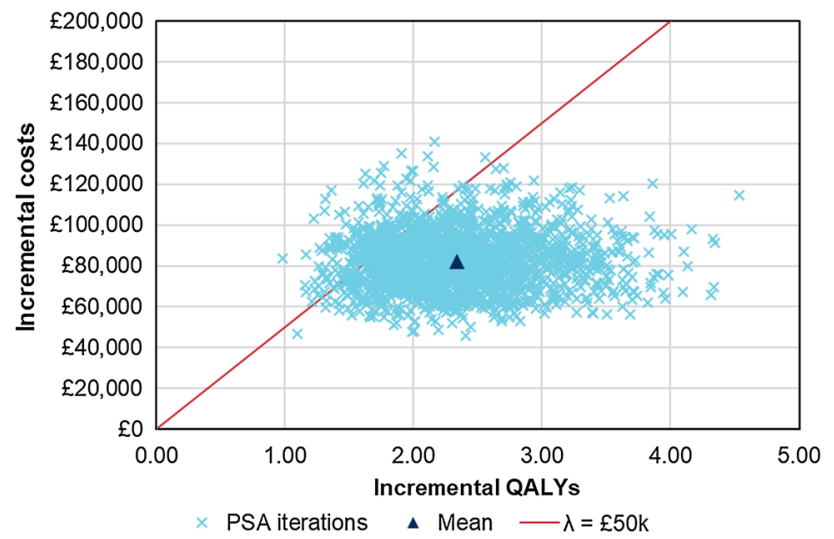

Fig. 6 Probabilistic sensitivity analysis scatterplot versus standard care, treatment-experienced mMCC patients. $\lambda$ willingness-to-pay threshold, $m M C C$ metastatic Merkel cell carcinoma, PSA probabilistic sensitivity analysis, $Q A L Y s$ quality-adjusted life-years

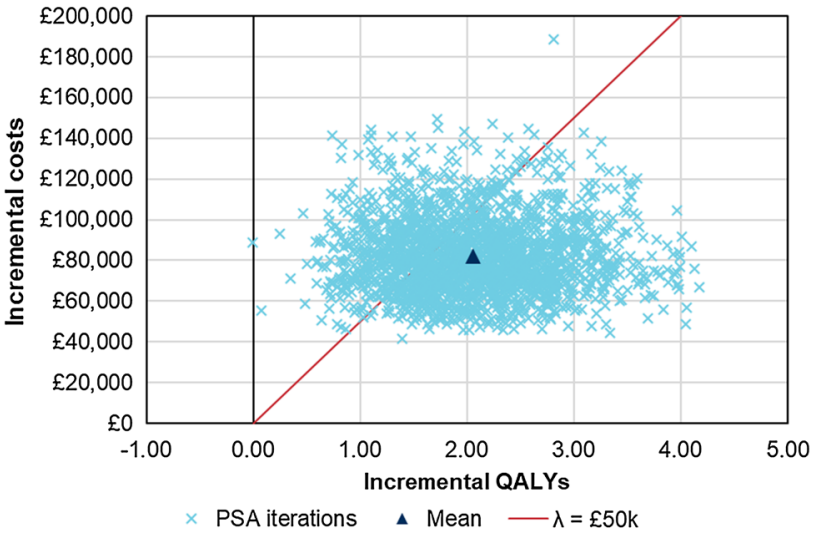

Fig. 7 Probabilistic sensitivity analysis scatterplot versus standard care, treatment-naive mMCC patients. $\lambda$ willingness-to-pay threshold, $m M C C$ metastatic Merkel cell carcinoma, PSA probabilistic sensitivity analysis, $Q A L Y s$ quality-adjusted life-years

methods of direct and indirect comparison will remain a limitation. Extensive data collection through the generation of data for the SC arm and clinical expert validation was undertaken to mitigate this area of uncertainty within the CEA.

While data from Part A of the JAVELIN Merkel 200 trial (TE patients) are sufficiently mature to generate robust estimates of the cost effectiveness of avelumab, data from Part $\mathrm{B}$ (TN patients) are still maturing. The CEA results for TN patients in this study provide indicative cost-effectiveness estimates for avelumab, with extended follow-up needed from Part B of the JAVELIN Merkel 200 trial to establish the full benefits of avelumab in a first-line setting. However, these early CEA results indicate avelumab is likely to be a cost-effective treatment option for $\mathrm{TN}$ patients based on clinically validated CEA inputs and assumptions based on the mature TE data.

\section{Conclusions}

Our study exemplifies avelumab as a promising, innovative and cost-effective treatment for a small, underserved patient population with limited, and unlicensed, treatment options associated with a poor benefit-to-risk ratio. In May 2018, the availability of avelumab extended to include all UK TE and TN patients with mMCC in England and Scotland [18, 58]. Avelumab therefore represents a step change in therapy to these patients, and a cost-effective use of NHS resources with a limited budget impact based on an incident population of approximately $100 \mathrm{UK} \mathrm{mMCC}$ patients per year.

Acknowledgements The authors thank the patients and their families, investigators, co-investigators, and study teams at each of the participating centres at Merck KGaA, Darmstadt, Germany, and EMD Serono, Billerica, MA, USA (a business of Merck KGaA, Darmstadt, Germany). 
Data Availability Statement The datasets generated and analysed during the current study are available from the corresponding author upon reasonable request.

Author contributions $\mathrm{AB}$ drafted the initial manuscript. $\mathrm{AB}$ and $\mathrm{AW}$ constructed the model. PN provided expert insight and clinically validated the model inputs and results. AH conducted supportive analyses to implement within the model. AA, CS, CP, MB, and CL reviewed the initial model and provided input into the overall structure and assumptions, as well as contributing to the original HTA submissions from which the model was based. Data for inclusion within the model were provided by AA, CS, CP, and MB via the pivotal clinical trial and supportive data collection exercises. All authors reviewed each version of the manuscript.

\section{Compliance with Ethical Standards}

Conflict of interest Murtuza Bharmal and Chris Pescott are employees of Merck KGaA, Darmstadt, Germany; Ceilidh Stapelkamp and Amerah Amin are employees of EMD Serono, Northwood, UK. At the time of the study, Ash Bullement and Anthony Hatswell were employees of BresMed, who were a paid consultant to Merck KGaA, Darmstadt, Germany. Anna Willis and Cameron Lilley are employees of BresMed, who were a paid consultant to Merck KGaA, Darmstadt, Germany. Paul Nathan received consulting fees for advisory boards for Merck $\mathrm{KGaA}$ and Pfizer.

Sources of funding This study was funded by and is part of an alliance between Merck KGaA and Pfizer, Inc., NY, USA.

Open Access This article is distributed under the terms of the Creative Commons Attribution-NonCommercial 4.0 International License (http://creativecommons.org/licenses/by-nc/4.0/), which permits any noncommercial use, distribution, and reproduction in any medium, provided you give appropriate credit to the original author(s) and the source, provide a link to the Creative Commons license, and indicate if changes were made.

\section{References}

1. Grabowski J, Saltzstein SL, Sadler GR, Tahir Z, Blair S. A comparison of merkel cell carcinoma and melanoma: results from the california cancer registry. Clin Med Oncol. 2008;2:327-33.

2. Goon PK, Greenberg DC, Igali L, Levell NJ. Merkel cell carcinoma: rising incidence in the East of England. J Eur Acad Dermatol Venereol. 2016;30(12):2052-5.

3. Tello TL, Coggshall K, Yom SS, Yu SS. Merkel cell carcinoma: an update and review: current and future therapy. J Am Acad Dermatol. 2018;78(3):445-54.

4. National Cancer Intelligence Network (NCIN). Rare skin cancer in England: NCIN data briefing. 2011. http://www.ncin.org.uk/publi cations/data_briefings/rareskincancer. Accessed 4 May 2018.

5. Lebbe C, Becker JC, Grob JJ, Malvehy J, Del Marmol V, Pehamberger H, et al. Diagnosis and treatment of Merkel cell. Carcinoma European consensus-based interdisciplinary guideline. Eur J Cancer. 2015;51(16):2396-403.

6. Coggshall K, Tello TL, North JP, Yu SS. Merkel cell carcinoma: an update and review: pathogenesis, diagnosis, and staging. J Am Acad Dermatol. 2018;78(3):433-42.

7. Kaufman HL, Russell J, Hamid O, Bhatia S, Terheyden P, D'Angelo SP, et al. Avelumab in patients with chemotherapy-refractory metastatic Merkel cell carcinoma: a multicentre, single-group, open-label, phase 2 trial. Lancet Oncol. 2016;17(10):1374-85.

8. Santamaria-Barria JA, Boland GM, Yeap BY, Nardi V, Dias-Santagata D, Cusack JC Jr. Merkel cell carcinoma: 30-year experience from a single institution. Ann Surg Oncol. 2013;20(4):1365-73.

9. Allen PJ, Bowne WB, Jaques DP, Brennan MF, Busam K, Coit DG. Merkel cell carcinoma: prognosis and treatment of patients from a single institution. J Clin Oncol. 2005;23(10):2300-9.

10. Stokes JB, Graw KS, Dengel LT, Swenson BR, Bauer TW, Slingluff $\mathrm{CL}$, et al. Patients with merkel cell carcinoma tumors $\leq 1.0 \mathrm{~cm}$ in diameter are unlikely to harbor regional lymph node metastasis. J Clin Oncol. 2009;27(23):3772-7.

11. Anand G. London cancer: skin cancer radiotherapy guidelines. 2013. http://www.londoncancer.org/media/76382/london-cance r-skin-radiotherapy-guidelines-2013-v1.0.pdf. Accessed 4 Apr 2018 (updated August 2014).

12. Iyer JG, Blom A, Doumani R, Lewis C, Tarabadkar ES, Anderson A, et al. Response rates and durability of chemotherapy among 62 patients with metastatic Merkel cell carcinoma. Cancer Med. 2016;5(9):2294-301.

13. Voog E, Biron P, Martin JP, Blay JY. Chemotherapy for patients with locally advanced or metastatic Merkel cell carcinoma. Cancer. 1999;85(12):2589-95.

14. Merck Serono Europe Limited. Avelumab (BAVENCIO). Summary of product characteristics (SPC). 2017. https://www.ema. europa.eu/documents/product-information/bavencio-epar-produ ctinformation_en.pdf. Accessed 14 Jan 2019.

15. Clinicaltrials.gov. Avelumab in subjects with merkel cell carcinoma (JAVELIN Merkel 200). 2018. https://clinicaltrials.gov/ct2/ show/record/NCT02155647. Accessed 4 May 2018 (updated 12 April 2018).

16. D'Angelo SP, Russell J, Lebbe C, Chmielowski B, Gambichler $\mathrm{T}$, Grob JJ, et al. Efficacy and safety of first-line avelumab treatment in patients with stage IV metastatic Merkel cell carcinoma: a preplanned interim analysis of a clinical trial. JAMA Oncol. 2018;4(9):e180077.

17. National Institute for Health and Care Excellence (NICE). Final scope: avelumab for treating metastatic Merkel cell carcinoma. 2017. https://www.nice.org.uk/guidance/ta517/documents/final -scope. Accessed 4 May 2018 (updated March 2017).

18. National Institute for Health and Care Excellence (NICE). Final appraisal determination: avelumab for treating metastatic Merkel cell carcinoma. 2018. https://www.nice.org.uk/guidance/ta517/ documents/final-appraisal-determination-document. Accessed 4 May 2018.

19. Nghiem P, Shailender B, Scott Brohl A, Hamid O, Mehnert JM, Terheyden $\mathrm{P}$, et al. Two-year efficacy and safety update from JAVELIN Merkel 200 part A: a registrational study of avelumab in metastatic Merkel cell carcinoma progressed on chemotherapy. J Clin Oncol. 2018;36(15 Suppl):9507.

20. Ball G, Xie F, Tarride J-E. Economic evaluation of bevacizumab for treatment of platinum-resistant recurrent ovarian cancer in Canada. PharmacoEconomics Open. 2018;2(1):19-29.

21. Bullement A, Underhill S, Fougeray R, Hatswell AJ. Cost-effectiveness of trifluridine/tipiracil for previously treated metastatic colorectal cancer in England and Wales. Clin Colorectal Cancer. 2018;17(1):e143-51.

22. McCrea C, Johal S, Yang S, Doan J. Cost-effectiveness of nivolumab in patients with advanced renal cell carcinoma treated in the United States. Exp Hematol Oncol. 2018;7:4.

23. Huang M, Lou Y, Pellissier J, Burke T, Liu FX, Xu R, et al. Cost effectiveness of pembrolizumab vs. standard-of-care chemotherapy as first-line treatment for metastatic NSCLC that expresses 
high levels of PD-L1 in the United States. PharmacoEconomics. 2017;35(8):831-44.

24. National Institute for Health and Care Excellence (NICE). Guide to the methods of technology appraisal. 2013. https://www.nice. org.uk/process/pmg9/resources/guide-to-the-methods-of-techn ology-appraisal-2013-pdf-2007975843781. Accessed Apr 2017.

25. Merck KGaA. Consultant Oncologist. Avelumab in metastatic Merkel cell carcinoma, validation meeting report; 2017.

26. Cowey CL, Mahnke L, Espirito J, Helwig C, Oksen D, Bharmal M. Real-world treatment outcomes in patients with metastatic Merkel cell carcinoma treated with chemotherapy in the USA. Future oncology. 2017;13(19):1699-710.

27. Cowey CL, Becker J, Bharmal M. Retrospective observational study to evaluate treatment outcomes in patients with metastatic merkel cell carcinoma following chemotherapy. Clinical study report; 2016.

28. Becker J, Lorenz E, Haas G, Helwig C, Oksen D, Mahnke L, et al. Evaluation of real world treatment outcomes in patients with metastatic merkel cell carcinoma (MCC) following second line chemotherapy. Ann Oncol. 2016;27(Suppl 6):1154P-P.

29. Samlowski WE, Moon J, Tuthill RJ, Heinrich MC, Balzer-Haas NS, Merl SA, et al. A phase II trial of imatinib mesylate in merkel cell carcinoma (neuroendocrine carcinoma of the skin): a Southwest Oncology Group study (S0331). Am J Clin Oncol. 2010;33(5):495-9.

30. Fields RC, Busam KJ, Chou JF, Panageas KS, Pulitzer MP, Allen PJ, et al. Five hundred patients with Merkel cell carcinoma evaluated at a single institution. Ann Surg. 2011;254(3):465-73 (discussion 73-5).

31. Edwards SJ, Wakefield V, Cain P, Kew KM, Salih F. Avelumab for treating metastatic Merkel cell carcinoma: a single technology appraisal. BMJ Technology Assessment Group. 2017. https://www.nice.org.uk/guidance/ta517/documents/committeepapers. Accessed 10 May 2018.

32. Hatswell AJ, Cooper M, Bharmal M, Bullement A. Predicting outcomes from multiple uncontrolled historical studies of treatment of physicians choice: progression-free survival and overall survival in untreated metastatic Merkel cell carcinoma. Value Health. 2017;20(9):A422.

33. Merck KGaA. Company submission: avelumab for treating metastatic Merkel cell carcinoma. 2017. https://www.nice.org. uk/guidance/ta517/documents/committee-papers. Accessed 4 Jun 2018 (updated 22 February 2018).

34. Latimer N. NICE DSU technical support document 14: survival analysis. NICE; 2011.

35. Royston P, Parmar MK. Flexible parametric proportionalhazards and proportional-odds models for censored survival data, with application to prognostic modelling and estimation of treatment effects. Stat Med. 2002;21(15):2175-97.

36. Socinski MA, Smit EF, Lorigan P, Konduri K, Reck M, Szczesna A, et al. Phase III study of pemetrexed plus carboplatin compared with etoposide plus carboplatin in chemotherapynaive patients with extensive-stage small-cell lung cancer. J Clin Oncol. 2009;27(28):4787-92.

37. Sun Y, Cheng Y, Hao X, Wang J, Hu C, Han B, et al. Randomized phase III trial of amrubicin/cisplatin versus etoposide/ cisplatin as first-line treatment for extensive small-cell lung cancer. BMC Cancer. 2016;16:265.

38. Herdman M, Gudex C, Lloyd A, Janssen MF, Kind P, Parkin $\mathrm{D}$, et al. Development and preliminary testing of the new five-level version of EQ-5D (EQ-5D-5L). Qual Life Res. 2011;20(10):1727-36.

39. Kaufman HL, Hunger M, Hennessy M, Schlichting M, Bharmal M. Nonprogression with avelumab treatment associated with gains in quality of life in metastatic Merkel cell carcinoma. Future Oncol. 2018;14(3):255-66.
40. Hatswell AJ, Pennington B, Pericleous L, Rowen D, Lebmeier M, Lee D. Patient-reported utilities in advanced or metastatic melanoma, including analysis of utilities by time to death. Health Qual Life Outcomes. 2014;12:140.

41. van Hout B, Janssen MF, Feng YS, Kohlmann T, Busschbach J, Golicki D, et al. Interim scoring for the EQ-5D-5L: mapping the EQ-5D-5L to EQ-5D-3L value sets. Value Health. 2012;15(5):708-15.

42. Devlin N, Shah K, Feng Y, Mulhern B, van Hout B. Valuing health-related quality of life: an EQ-5D-5L value set for England. London: Office of Health Economics; 2016.

43. Nafees B, Stafford M, Gavriel S, Bhalla S, Watkins J. Health state utilities for non small cell lung cancer. Health Qual Life Outcomes. 2008;6:84.

44. Tolley K, Goad C, Yi Y, Maroudas P, Haiderali A, Thompson G. Utility elicitation study in the UK general public for late-stage chronic lymphocytic leukaemia. Eur J Health Econ. 2013;14(5):749-59.

45. Ossa DF, Briggs A, McIntosh E, Cowell W, Littlewood T, Sculpher M. Recombinant erythropoietin for chemotherapy-related anaemia: economic value and health-related quality-of-life assessment using direct utility elicitation and discrete choice experiment methods. PharmacoEconomics. 2007;25(3):223-37.

46. British National Formulary (BNF). Avelumab solution for infusion. 2018. https://bnf.nice.org.uk/medicinal-forms/avelumab. html. Accessed 10 May 2018.

47. Merck KGaA. Patient level data: avelumab in patients with chemotherapy-refractory metastatic Merkel cell carcinoma: a multicentre, single-group, open-label, phase 2 trial. Darmstadt: Merck $\mathrm{KGaA} ; 2016$.

48. Hatswell AJ, Porter J, Hertel N, Lee D. The cost of costing treatments incorrectly: errors in the application of drug prices in economic models due to differing patient weights. Value. 2014;17(7):A323-4.

49. National Health Service England (NHSE). National dose banding table —avelumab. 2018. https://www.england.nhs.uk/wp-conte nt/uploads/2018/01/national-tables-avelumab-20mgmL-v2.pdf. Accessed 10 May 2018 (updated 19 April 2018).

50. Department of Health. NHS reference costs. 2016. https://www. gov.uk/government/collections/nhs-reference-costs. Accessed 24 Apr 2017.

51. Curtis L, Burns A. Unit costs of health and social care. University of Kent: Personal Social Services Research Unit; 2016.

52. Vouk K, Benter U, Amonkar MM, Marocco A, Stapelkamp C, Pfersch S, et al. Cost and economic burden of adverse events associated with metastatic melanoma treatments in five countries. $\mathrm{J}$ Med Econ. 2016;19(9):900-12.

53. Wehler E, Zhao Z, Pinar Bilir S, Munakata J, Barber B. Economic burden of toxicities associated with treating metastatic melanoma in eight countries. Eur J Health Econ. 2017;18(1):49-58.

54. Round J, Jones L, Morris S. Estimating the cost of caring for people with cancer at the end of life: a modelling study. Palliat Med. 2015;29(10):899-907.

55. National Institute for Health and Care Excellence (NICE). TA517: avelumab for treating metastatic Merkel cell carcinoma. 2018. https://www.nice.org.uk/guidance/ta517/documents/committeepapers. Accessed 24 Jul 2018.

56. Paulden M. Recent amendments to NICE's value-based assessment of health technologies: implicitly inequitable? Expert Rev Pharmacoecon Outcomes Res. 2017;17(3):239-42.

57. Uchi H. Merkel Cell carcinoma: an update and immunotherapy. Front Oncol. 2018;8:48.

58. Scottish Medicines Consortium (SMC). Detailed advice document (DAD): avelumab $20 \mathrm{mg} / \mathrm{mL}$ concentrate for solution for infusion (Bavencio ${ }^{\circledR}$ ) SMC no. 1315/18. 2018. https://www.scott ishmedicines.org.uk/media/3410/avelumab-bavencio-final-april 
-2018-for-website.pdf. Accessed 5 Jun 2018 (updated 7 May 2018).

59. National Institute for Health and Care Excellence. Guide to the methods of technology appraisal. 2013. https://www.nice.org.uk/ process/pmg9/chapter/the-reference-case. Accessed 24 Apr 2017.
60. Department of Health. Drugs and pharmaceutical electronic market information (eMit). 2016. https://www.gov.uk/government/ publications/drugs-and-pharmaceutical-electronic-market-infor mation-emit. Accessed 24 Apr 2017. 